Copyright by the Acoustical Society of America. Cho, Y. T. \& Roan, M. J. (2009). Adaptive near-field beamforming techniques for sound source imaging. Journal of the

Acoustical Society of America, 125(2), 944-957. doi: 10.1121/1.3050248

\title{
Adaptive near-field beamforming techniques for sound source imaging
}

\author{
Yong Thung $\mathrm{Cho}^{\mathrm{a})}$ and Michael J. Roan \\ Department of Mechanical Engineering, Virginia Polytech Institute and State University, \\ Blacksburg, Virginia 24061
}

(Received 5 May 2008; revised 4 November 2008; accepted 7 November 2008)

\begin{abstract}
Phased array signal processing techniques such as beamforming have a long history in applications such as sonar for detection and localization of far-field sound sources. Two sometimes competing challenges arise in any type of spatial processing; these are to minimize contributions from directions other than the look direction and minimize the width of the main lobe. To tackle this problem a large body of work has been devoted to the development of adaptive procedures that attempt to minimize side lobe contributions to the spatial processor output. In this paper, two adaptive beamforming procedures_minimum variance distorsionless response and weight optimization to minimize maximum side lobes-are modified for use in source visualization applications to estimate beamforming pressure and intensity using near-field pressure measurements. These adaptive techniques are compared to a fixed near-field focusing technique (both techniques use near-field beamforming weightings focusing at source locations estimated based on spherical wave array manifold vectors with spatial windows). Sound source resolution accuracies of near-field imaging procedures with different weighting strategies are compared using numerical simulations both in anechoic and reverberant environments with random measurement noise. Also, experimental results are given for near-field sound pressure measurements of an enclosed loudspeaker. (C) 2009 Acoustical Society of America. [DOI: 10.1121/1.3050248]
\end{abstract}

PACS number(s): 43.60.Fg, 43.60.Jn, 43.60.Lq, 43.60.Mn [EJS] Pages: 944-957

\section{INTRODUCTION}

Beamforming has a long history of use in applications such as sonar for detection and localization of far-field sound sources. ${ }^{1,2}$ For sources that lie in the far-field, beamforming has two primary uses: first, determining the direction to the source and, second, enhancing the signal-to-noise ratio. With modification, standard beamforming procedures can be used for near-field sound source imaging. Traditional far-field delay-and-sum beamforming can be modified to give good imaging performance in the near-field by using beamforming weights that are inversely proportional to the distance from the source to the measurement locations. Previous work has shown the utility in applying standard beamforming techniques to sound source identification. It has been used to reduce the effect of wind noise on sound measurements in a wind tunnel using an array of microphones. ${ }^{3}$ Also, the characteristics of jet noise sources were identified using beamformed far-field measurements. ${ }^{4}$ Passby noise from a vehicle has been measured in far-field and visualized using beamforming. ${ }^{5}$ The beamforming weighting was estimated using a maximum likelihood estimation of the amplitude of a single spherical source with additive white noise, which resulted in a weighting inversely proportional to the distance from the hypothesized source location to the measurement point. $^{5}$

A large body of work has been devoted to the development of far-field adaptive beamforming procedures with

\footnotetext{
a) Author to whom correspondence should be addressed. Electronic mail: cho.yong@gmail.com
}

the goal of reducing side lobe contributions to the beamformer output. ${ }^{1,6-10}$ Reducing side lobes improves source resolution accuracy for measurements made with and without reverberation. For an equally spaced linear array, it is possible to analytically find weights that minimize side lobe level or main lobe beam width using far-field pressure measurements. ${ }^{6}$ The minimum variance distorsionless response (MVDR) beamformer is one of the most widely used adaptive beamforming procedures and improves source resolution accuracy by adaptively finding the weights that minimize the output noise variance due to signals that arrive from directions other than the hypothesized source direction. ${ }^{1,7,8}$ The side lobe level of beamformed pressure can also be reduced by finding weights to minimize the maximum side lobes using an optimization procedure. ${ }^{9,10}$

The conventional delay-and-sum beamforming procedure can be modified to visualize sound sources based on near-field measurements. ${ }^{11}$ This is accomplished by modifying the form of the conventional beamforming ( $\mathrm{CBF}$ ) weights such that the beamformer focuses at specific points between the face of the array and the source rather than by steering the beamformer to coherently sum source contributions from a given direction. The weighting used to accomplish distance specific focusing is inversely proportional to the distance from the source to the measurement points. This reduces the weighting of measurements farther away from the source. This weighting method significantly improves the source resolution accuracy of the beamforming procedure based on near-field measurements.

In this work, two adaptive beamforming procedures: MVDR and optimized weights to minimize the maximum 


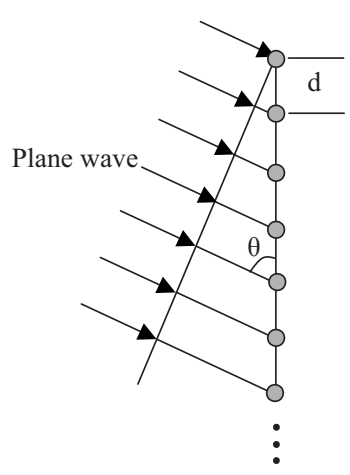

(b)

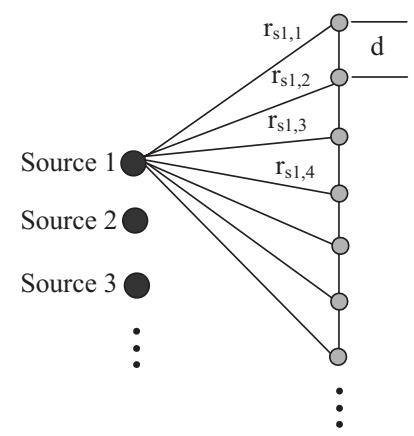

FIG. 1. Beamforming of measurement pressure in line array. (a) Plane wave source; (b) point sources.

side lobes (from here on referred to as optimized weights) are modified to perform acoustic source imaging based on near-field measurements. In addition, a weighting scheme is implemented where the weights are inversely proportional to higher orders of the distance from the hypothesized source to the measurement points. Near-field beamforming weights focusing at source locations are estimated based on spherical wave array manifold vectors with spatial windows. Numerical simulations compare the sound source resolution accuracies of the various weighting strategies. Multipole simulations compare the relative performance of the various weighting strategies when estimating beamformed intensity from near-field pressure measurements. The effects of random measurement noise and reverberation are quantified via appropriate simulations. In addition, near-field sound pressure of an enclosed loudspeaker was anechoically measured, and beamforming intensity estimates using various near-field beamforming procedures were compared.

\section{SOUND SOURCE IMAGING USING HIGH- RESOLUTION NEAR-FIELD BEAMFORMING}

This section introduces three high-resolution beamforming procedures for sound source imaging using near-field microphone measurements. In order to have good source resolution accuracy when using beamforming techniques, two criteria should be met: (1) the beamformer main lobe should be as narrow as possible and (2) side lobes should be as low as possible. The three high-resolution beamforming procedures introduced in this section each attempt to satisfy these criteria. The first technique uses weights that are inversely proportional to higher orders of the distance from the source to the measurement points. The net effect of this weighting scheme is that the beamformer focuses at points between the face of the array and the source. This reduces the contributions from all other points when reconstructing the source image. Second, an adaptive beamforming procedure, nearfield MVDR, is introduced to minimize side lobe contributions by adaptively placing nulls in the direction of sources other than the steering directions. Lastly a procedure is introduced that optimizes weights to minimize the maximum side lobes. The accuracies of the latter methods are compared in Secs. III and IV using simulations and measurements of an enclosed loudspeaker in an anechoic chamber.

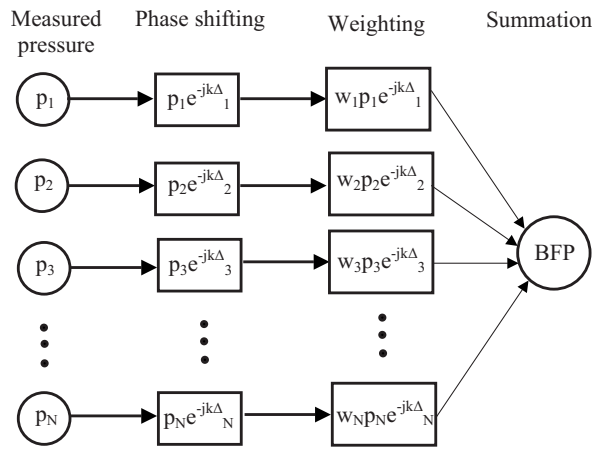

FIG. 2. Beamforming algorithm of measurement pressure

\section{A. Near-field beamforming for sound source imaging}

Beamforming is an effective technique to image sound sources using near-field measurements of the sound pressure field. This subsection provides the necessary background on the near-field beamforming technique.

The wave propagation from the sound source and the pressure measurement geometry are shown in Fig. 1. The beamforming algorithm for measurement pressure in the frequency domain is shown in Fig. 2. First, pressure is measured with an array of microphones, and the phase of the measurement pressure is shifted to account for the delay of wave propagation due to the difference in path length between microphones. Then, the weights are multiplied with the phase shifted measurement pressure and summed to estimate beamforming pressure for a given steering angle $\theta$ and frequency $\omega$.

By focusing the beamformer at a point instead of a certain direction $\theta$, as shown in Fig. 1(b), the locations of sources can be identified. Assuming $e^{-j \omega t}$ time convention, beamforming pressure focused on source $j, \mathrm{BFP}_{j}$, can be represented as

$$
\begin{aligned}
\mathrm{BFP}_{j}= & w_{j, 1} p_{1} e^{-j k \Delta_{j, 1}}+w_{j, 2} p_{2} e^{-j k \Delta_{j, 2}}+w_{j, 3} p_{3} e^{-j k \Delta_{j, 3}} \\
& +\cdots+w_{j, N} p_{N} e^{-j k \Delta_{j, N}} .
\end{aligned}
$$

The delay of the path length of pressure measured in each microphone focusing at source $j, \Delta_{j, i}$, is

$$
\Delta_{j, i}=r_{s j, i}
$$

where $r_{s j, i}$ is the distance from the hypothesized source $j$ to measurement location $i$. Weightings $w_{j, i}$ for uniform weighting is

$$
w_{j, i}=\frac{1}{N},
$$

which is simply averaging the phase shifted measurement pressure. However, if weightings inversely proportional to distance from source to measurement location are assumed, then

$$
w_{j, i}=\frac{1}{\Delta_{j, i} N}=\frac{1}{r_{s j, i} N} .
$$


Normalizing the weights with respect to distance,

$$
w_{j, i}=\frac{1}{r_{s j, i} \sum_{k=1}^{N} \frac{1}{r_{s j, k}}},
$$

ensures that the sum of the weights is 1 as for uniform weighting [Eq. (3)].

Beamforming pressure in Eq. (1) can also be applied to a two-dimensional array, and beamforming pressure can be estimated on the source surface. This process is similar to the back-projection procedure of acoustical holography. ${ }^{12-17}$ Since beamforming pressure can be estimated on an infinite number of surfaces close to the source, the beamforming particle velocity on the source surface can be found using Euler's equation. Also beamforming intensity on the source surface can be calculated from beamforming pressure and particle velocity estimated on the source surface.

More generally, anechoic, noise-free sound pressure $\mathbf{p}$ due to source signal $\mathbf{s}$, can be represented as

$$
\mathbf{p}=\mathbf{s v},
$$

where $\mathbf{v}$ is an array manifold vector, which is a transfer function between source signals and measurement pressure,

$$
\mathbf{v}=\left[\begin{array}{lllll}
A_{1} e^{j \phi_{1}}, & A_{2} e^{j \phi_{2}}, & A_{3} e^{j \phi_{3}}, & \ldots, & A_{N} e^{j \phi_{N}}
\end{array}\right],
$$

where $A_{i}$ 's and $\phi_{i}$ 's are the amplitude and phase of transfer function between the source and the measurement pressure. Source signal $\mathbf{s}$ can be estimated from measurement pressure p by multiplication of weight vector with phase compensation, w, as

$$
\mathbf{s}=\mathbf{p} \mathbf{w}^{T} .
$$

By postmultiplying $\mathbf{v}$ at both sides of Eq. (8) and is compared with Eq. (6),

$$
\mathbf{p}=\mathbf{s v}=\mathbf{p w}^{T} \mathbf{v}
$$

under the constraint ${ }^{7}$

$$
\mathbf{w}^{T} \mathbf{v}=I,
$$

where $I$ is an identity matrix. The relationship in Eq. (10) is true regardless of the manifold vector or the source. By postmultiplying $\mathbf{v}^{H}$ at both sides of Eq. (10) and dividing by $\mathbf{v} \mathbf{v}^{H}$, which is a constant, the weight vector is estimated as

$$
\mathbf{w}^{T}=\mathbf{v}^{H} / \mathbf{v} \mathbf{v}^{H} .
$$

The weight vector estimate for anechoic and noise-free pressure measurement in Eq. (11) is a normalized complex conjugate of the array manifold vector and is independent of source signals.

By assuming spherical wave from the source, the array manifold vector is represented as

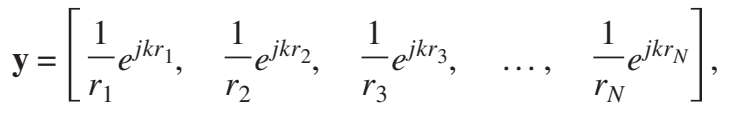

where $r_{i}$ 's represent the distance from the source to the measurement location. By substituting the array manifold vector in Eq. (12) into the weight vector in Eq. (11), amplitude weights are

$$
w_{i}=\frac{1}{r_{i} \sum_{k=1}^{N} \frac{1}{r_{k}^{2}}},
$$

which is identical to the amplitude of weights presented in Eq. (5) except for the normalization of the amplitude of the weights. ${ }^{5}$ Therefore, beamforming weights whose magnitudes are inversely proportional to the distance between the source and the measurement location are obtained by assuming spherical waves propagating from the source in an anechoic, noise-free environment.

\section{B. High order inversely proportional beamforming}

Inversely proportional beamforming (IWBF) weights are derived for spherical waves propagating from the source in an anechoic, noise-free environment, as shown in Sec. II A. For more general cases, sound radiation in the radial direction from motion of a sphere can be represented by a combination of spherical Hankel function of order $m$. For relatively large $k r$, e.g., $k r>10$, the spherical Hankel function of order $m$ converges to spherical waves (spherical Hankel function of order $m=0$ ). The latter implies that IWBF is more accurate for larger $k r$ or at higher frequencies. However, for the smaller $k r$ or at lower frequencies, spherical Hankel functions of higher order $m$ decay more rapidly than $m=0$ as $k r$ increases. The latter property of the spherical Hankel function causes the measurement pressure at smaller $k r$ or at lower frequencies containing high order components to drop rapidly below the noise floor. The higher order measurement pressure is dominated by measurement noise as the measurement is taken farther away from the source. However, measurement pressure taken farther away from the source, especially at low frequencies, possibly corrupted by measurement noise can be filtered during the beamforming process by implementing high order IWBF.

A significantly higher resolution of the source can be obtained using weightings that are inversely proportional to higher orders of the distance from source to measurement location rather than uniform weightings. If higher order weightings inversely proportional to distance from source to measurement location are assumed, the weightings are

$$
w_{j, i}=\frac{1}{\Delta_{j, i}^{n} N}=\frac{1}{r_{s j, i}^{n} N},
$$

where $n$ is the order of inversely proportional weighting. Normalizing the higher order weights with respect to distance gives

$$
w_{j, i}=\frac{1}{r_{s j, i}^{n} \sum_{k=1}^{N} \frac{1}{r_{s j, k}^{n}}} .
$$

This ensures that the sum of the weights is 1 as for uniform weighting [Eq. (3)].

Beamforming weights shown in Eqs. (5), (14), and (15) can also be represented as spatial windows applied to mea- 

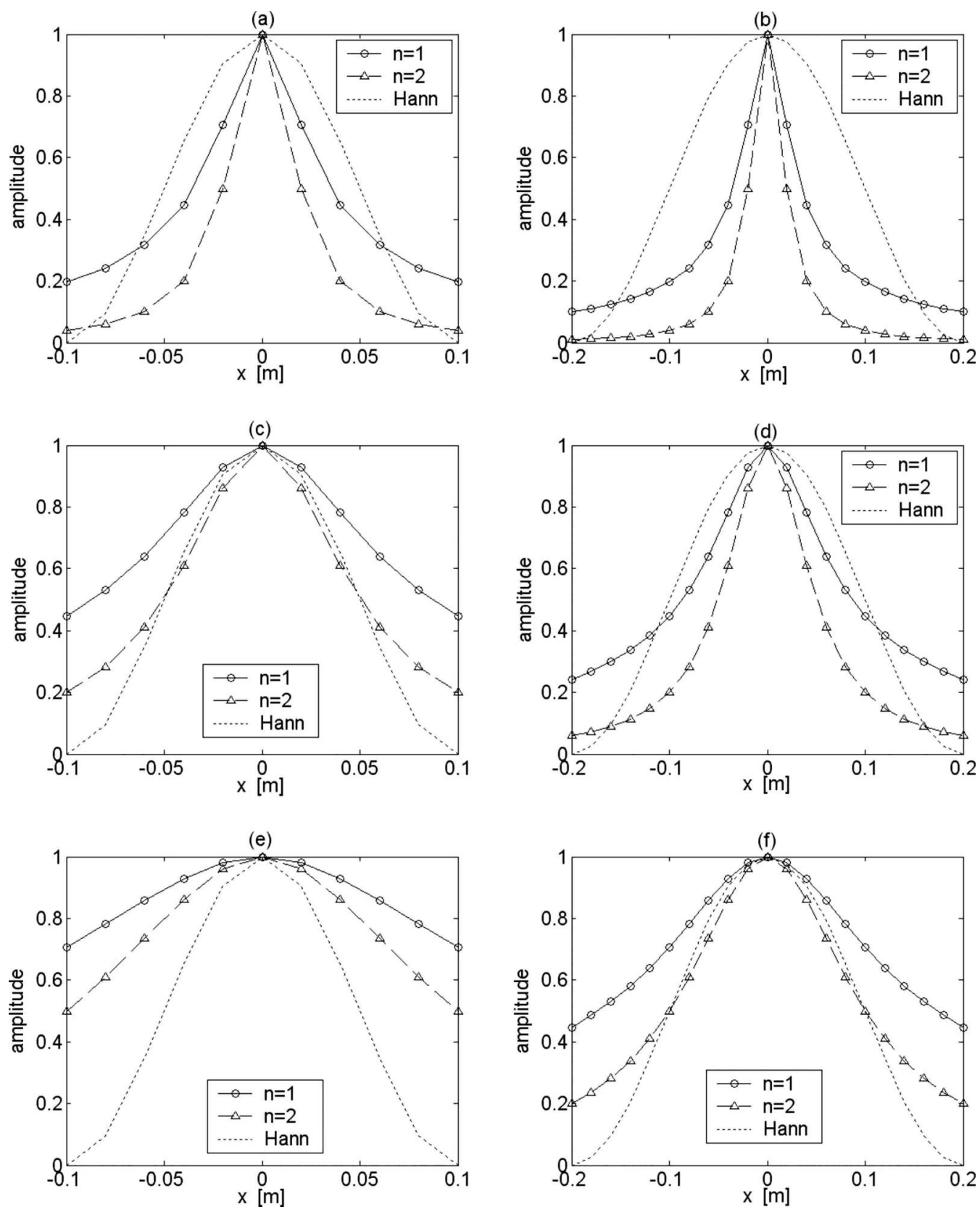

FIG. 3. Normalized amplitude of IWBF window and Hanning window applied to measurement pressure with various measurement locations and measurement aperture sizes. (a) $L_{x}=0.2 \mathrm{~m}, z=0.02 \mathrm{~m}$; (b) $L_{x}=0.4 \mathrm{~m}, z=0.02 \mathrm{~m}$; (c) $L_{x}=0.2 \mathrm{~m}, z=0.05 \mathrm{~m}$; (d) $L_{x}=0.4 \mathrm{~m}, z=0.05 \mathrm{~m}$; (e) $L_{x}=0.2 \mathrm{~m}, z=0.1 \mathrm{~m}$; (f) $L_{x}$ $=0.4 \mathrm{~m}, z=0.1 \mathrm{~m}$.

surement pressure. Normalized amplitudes of the IWBF and Hanning windows applied to measurement pressure with various measurement locations and measurement sizes are shown in Fig. 3. The lengths of the linear measurement array, $L_{x}$, are 0.2 and $0.4 \mathrm{~m}$, and the spacing between measurement points is $2 \mathrm{~cm}$. A point source is supposed to be located at the coordinate origin, and the measurement array is located at $z=2 \mathrm{~cm}, z=5 \mathrm{~cm}$, and $z=10 \mathrm{~cm}$.

\section{Minimum variance distorsionless response beamformer}

In this section, the adaptive near-field MVDR beamforming procedure is derived to minimize contributions from sources that lie in directions other than the focusing point.
Near-field MVDR beamformer weights are derived to minimize the output noise power or maximize the array gain with a distortionless response constraint. ${ }^{1,7,8}$

First, measurement pressure can be represented as a superposition of the pressure directly radiated from source and measurement noise,

$$
\mathbf{p}=\mathbf{p}_{\mathrm{s}}+\mathbf{p}_{\mathbf{n}}
$$

where $\mathbf{p}_{\mathbf{s}}$ is the pressure directly radiated from source and $\mathbf{p}_{\mathbf{n}}$ is the measurement noise. The source property estimate, $\mathbf{s}_{\mathbf{n}}$, based on measurement pressure, $\mathbf{p}$, can be represented using a beamforming weight vector, which is, ${ }^{1}$ 


$$
\mathbf{S}_{\mathbf{n}}=\mathbf{p} \mathbf{w}^{T}
$$

Cross-power spectral matrix or variation of the source output estimate based on measurement pressure, $\mathbf{S}_{\mathbf{n}}$, is

$$
\mathbf{S}_{\mathbf{n}}=\mathbf{s}_{\mathbf{n}}^{H} \mathbf{S}_{\mathbf{n}}=\mathbf{w}^{*} \mathbf{P} \mathbf{w}^{T},
$$

where $\mathbf{P}$ is the cross-power spectral matrix of the measurement pressure with noise. To find the maximum value of the variance of the source output and the corresponding weights, a Lagrange multiplier was used, satisfying the distorsionless response relationship between the array manifold vector and weights, which is ${ }^{1}$

$$
\mathbf{v} \mathbf{w}^{T}=1
$$

The maximization function, $F$, is

$$
F=\mathbf{w}^{*} \mathbf{P} \mathbf{w}^{T}+\lambda\left(1-\mathbf{v} \mathbf{w}^{T}\right),
$$

where $\lambda$ is the Lagrange multiplier. By taking the derivative of the maximization function, $F$, with respect to the weight vector, $\mathbf{w}^{T}$, and if the derivative is zero when maximum,

$$
\frac{\partial F}{\partial \mathbf{w}^{T}}=\mathbf{w}^{*} \mathbf{P}-\lambda \mathbf{v}=0
$$

and $\mathbf{w}^{*}$ reduces to

$$
\mathbf{w}^{*}=\lambda \mathbf{v} \mathbf{P}^{-1} .
$$

By substituting Eq. (22) into the Hermitian of Eq. (19), the Lagrange multiplier, $\lambda$, is estimated as

$$
\lambda=\frac{1}{\mathbf{v} \mathbf{P}^{-1} \mathbf{v}^{H}} .
$$

By substituting Eq. (23) into Eq. (22), w* is estimated as

$$
\mathbf{w}^{*}=\frac{\mathbf{v} \mathbf{P}^{-1}}{\mathbf{v} \mathbf{P}^{-1} \mathbf{v}^{H}} \text {. }
$$

The weight vector derived in Eq. (24) maximizes the beamformed source output and is a function of the array manifold vector and cross-power spectrum of the measurement pressure. No specific wave type was assumed in the array manifold vector while deriving the weight vector except for the constraint between the weight and the array manifold vectors. There is no restriction about the source type when estimating the array manifold vector in Eq. (24). Either planar wave or spherical wave sources can be implemented when estimating the array manifold vector. Also spatial windows can be incorporated in the array manifold vector combined with spherical wave sources. As a result, measurement pressure taken farther away from the source especially at low frequencies possibly corrupted by measurement noise can be filtered during the beamforming process.

For a beamformer focusing at source $j, \mathbf{w}_{j}$ is the vector of weights with compensated phase, defined as

$$
\mathbf{w}_{j}=\left[w_{j, 1} e^{-j k \Delta_{j, 1}}, w_{j, 2} e^{-j k \Delta_{j, 2}}, w_{j, 3} e^{-j k \Delta_{j, 3}}, \ldots, w_{j, N} e^{-j k \Delta_{j, N}}\right] .
$$

The MVDR weight vector minimizing the output noise power or maximizing the array gain focusing at source $j$ is derived to be $\mathrm{e}^{1,7,8}$

$$
\mathbf{w}_{j}^{*}=\frac{\mathbf{v}_{j} \mathbf{P}^{-1}}{\mathbf{v}_{j} \mathbf{P}^{-1} \mathbf{v}_{j}^{H}}
$$

where $\mathbf{P}$ is a cross-power spectral matrix of measurement pressure. The cross-power spectral matrix of measurement pressure is estimated by the multiplication of Hermitian of the measurement pressure vector and the measurement pressure vector itself.

However, cross-power spectral matrix may be ill conditioned and requires regularization for estimating the inverse matrix. For a more accurate near-field implementation of MVDR, spatial filtering and spherical wave are incorporated in the array manifold vector. In order to have accurate nearfield focusing based on the MVDR algorithm, a new array manifold vector $\mathbf{X}_{j}$ with spatial filtering and spherical wave is introduced as

$$
\mathbf{X}_{j}=\left[\frac{e^{j k \Delta_{j, 1}}}{\mu_{j, 1}^{n}}, \frac{e^{j k \Delta_{j, 2}}}{\mu_{j, 2}^{n}}, \frac{e^{j k \Delta_{j, 3}}}{\mu_{j, 3}^{n}}, \ldots, \frac{e^{j k \Delta_{j, N}}}{\mu_{j, N}^{n}}\right],
$$

where $n-1$ is the order of the spatial window and $n=1$ represents a spherical wave with uniform window. The delay of the path length of pressure measured by each microphone is then focused on a surface between source $j$ and measurement surface, and $\mu_{j, i}$ is

$$
\mu_{j, i}=r_{f s j, i}
$$

where $r_{f s j, i}$ is the distance from point $j$ on surface between source and measurement surface to measurement location $i$. The amplitude of the near-field MVDR weight vector is

$$
\mathbf{A}_{j}=\left|\mathbf{X}_{j} \mathbf{P}^{-1} /\left(\mathbf{X}_{j} \mathbf{P}^{-1} \mathbf{X}_{j}^{H}\right)\right| \text {. }
$$

The normalized and phase corrected near-field MVDR weight vector is now given by

$$
\mathbf{w}_{j, i}=\mathbf{A}_{j, i} \mathbf{v}_{\mathbf{p} j, i}^{*} / \sum_{k=1}^{N} \mathbf{A}_{j, k},
$$

where $\mathbf{v}_{\mathbf{p}}$ is defined as

$$
\mathbf{v}_{\mathbf{p} j}=\left[e^{j k \Delta_{j, i}}, e^{j k \Delta_{j, 2}}, e^{j k \Delta_{j, 3}}, \ldots, e^{j k \Delta_{j, N}}\right],
$$

which is the plane wave array manifold vector focusing at source locations.

The weighting strategy given in Eq. (30) combines the amplitude weighting of MVDR with spherical wave and spatial filtering of measurement pressure and the phase information as used in standard frequency domain delay-and-sum beamforming to give robust adaptive near-field performance. The optimal location of the focusing surface for the calculation of MVDR weight amplitudes in Eq. (29) may or may not coincide with the hypothesized source location in Eq. (31), depending on the measurement geometry, projection distance, etc. This is because the shape of the spatial filter depends on both measurement geometry and projection distance, as shown in Fig. 3, and optimal spatial filtering depends on the source, measurement noise, etc. As the distance between the measurement and the source surfaces increases, the shape of the spatial filtering becomes relatively more 
uniform, as shown in Fig. 3. However, for both lower frequencies and signal-to-noise ratio of measurement pressure, a relatively sharper spatial window should be applied to the measurement pressure even though measurement surface is located farther away from the source surface. This can be accomplished by introducing hypothesized source location that is located closer to the measurement surface than on the actual source. The shape of the spatial window is influenced by the choice of hypothesized source location and the order of beamforming spatial window. This type of weighting significantly improves source resolution performance when using near-field measurements and is referred to as near-field MVDR in the present work.

\section{Optimization of weights to minimize the maximum side lobe level}

Beamforming with optimized array element position and weights to minimize both the number of elements in array and the maximum side lobe level of linear and sparse arrays was investigated extensively for various applications. ${ }^{18-26}$ Optimal weights to minimize main lobe width or maximum side lobe level for an equally spaced linear array can be analytically calculated using Dolph-Chebyshev array weighting. ${ }^{1,6}$ Optimal weights to minimize maximum side lobe level for both equally spaced and sparse linear and two-dimensional arrays can be estimated using linear programming. ${ }^{9,10}$

Similar to Holm's method, ${ }^{9,10}$ the maximum side lobe level for near-field beamforming pressure, that is, maximum beamforming pressure level except the main lobe region, can be minimized by finding the appropriate weighting using optimization. The normalized maximum side lobe level of nearfield beamforming pressure $\delta_{s}$ can be estimated from Eq. (1), excluding the main lobe region, as

$$
\begin{aligned}
\delta_{s}= & \frac{\max \left(\left|\mathrm{BFP}_{j}\right|\right)}{\left|\mathrm{BFP}_{\max }\right|} \\
& \text { (for } j=1,2, \cdots, N \text { except the main lobe region), }
\end{aligned}
$$

where $\mathrm{BFP}_{\max }$ represents the maximum value of $\mathrm{BFP}_{j}$ in the main lobe region. The optimal weights to minimize the maximum side lobe level $\delta_{s}$ are found using the constraint,

$$
\sum_{i=1}^{N} w_{j, i}=1 \quad(\text { for } j=1,2, \cdots, N) .
$$

However, in the present work, the weighting is supposed to be a function of only the distance between the focusing point and measurement location as

$$
w_{j, i}=f\left(r_{s j, i}\right),
$$

which remarkably reduces the number of optimal weightings to be estimated and reduces the required computation time by orders of magnitude especially when the number of measurements is large. Higher resolution near-field beamforming with optimized weights to minimize maximum side lobe level can be applied for equally spaced and sparse linear and

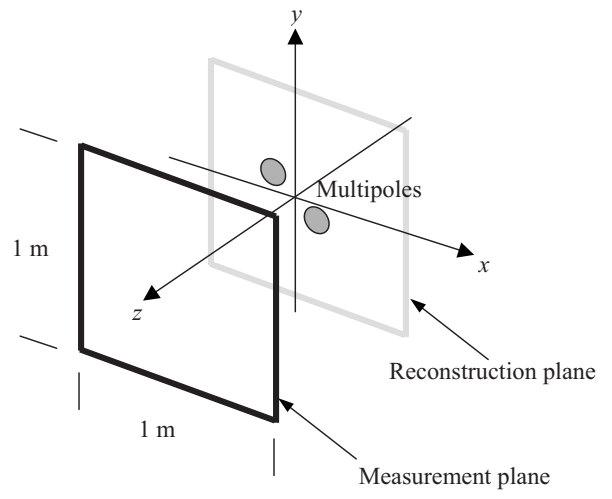

FIG. 4. Measurement geometry definition of multipole simulation with planar array.

two-dimensional arrays. Optimized weights to minimize the maximum side lobe level is referred to as optimized weights in the present work.

\section{NUMERICAL SIMULATIONS}

Several sets of numerical simulations of sound source imaging based on near-field acoustic measurements with two-dimensional arrays were performed to validate and compare the accuracy of the high-resolution near-field beamforming approaches. Details and results of the numerical simulations are described in this section.

First, anechoic sound fields radiated by out-of-plane multipoles separated by a distance smaller than wavelength were generated and measured numerically without measurement noise using a two-dimensional array at different frequencies and distances from the sources. In addition, reverberant sound fields radiated by the same multipoles were generated and measured numerically with random measurement noise using a two-dimensional array at different frequencies and distances from the sources. Normalized beamforming pressure and intensity were compared with those estimated using uniform weighting, higher order inversely proportional weighting, optimized weighting to minimize maximum side lobe level, and near-field MVDR weighting. Numerical simulations are described in more detail, and results are given in Secs. III A and III B.

\section{A. Anechoic multipole simulation with a two- dimensional array}

In this section, a multipole simulation is performed to compare the relative performance of near-field beamforming procedures with different weighting strategies for complicated sources.

The multipole simulations consist of the pressure field generated by ten monopoles. The measurement geometry of the multipole simulation with a $1 \mathrm{~m}$ square planar array is shown in Fig. 4. Measurement spacing is $5 \mathrm{~cm}$ both in $x$-and $y$-directions. Sound pressure is measured at $z=0.1 \mathrm{~m}$ and $z$ $=0.05 \mathrm{~m}$ for 1000 and $3000 \mathrm{~Hz}$, respectively. The location and amplitudes of monopoles that make up the multipoles are shown in Fig. 5. The centroids of the multipoles are 
(a)

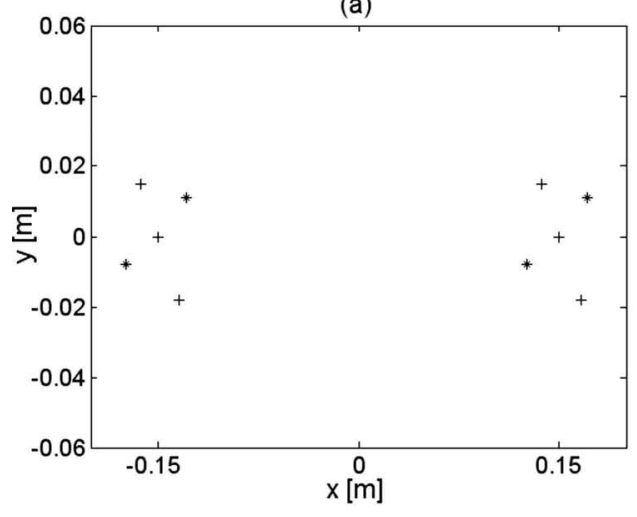

(c)

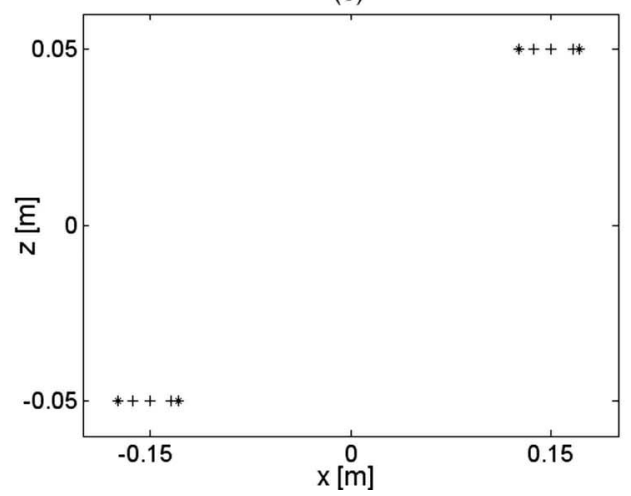

(b)

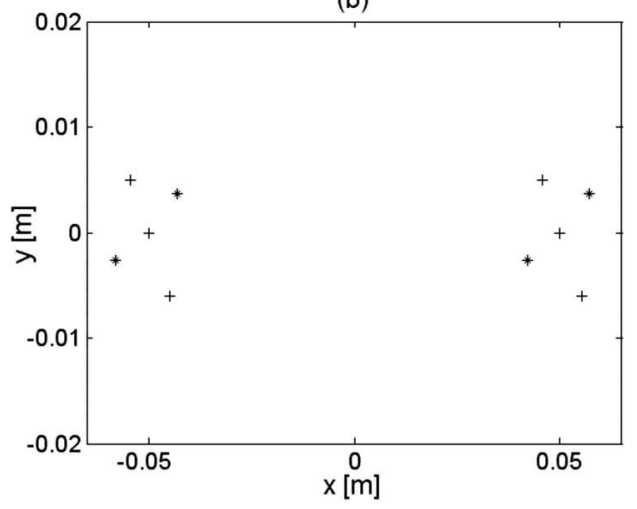

(d)

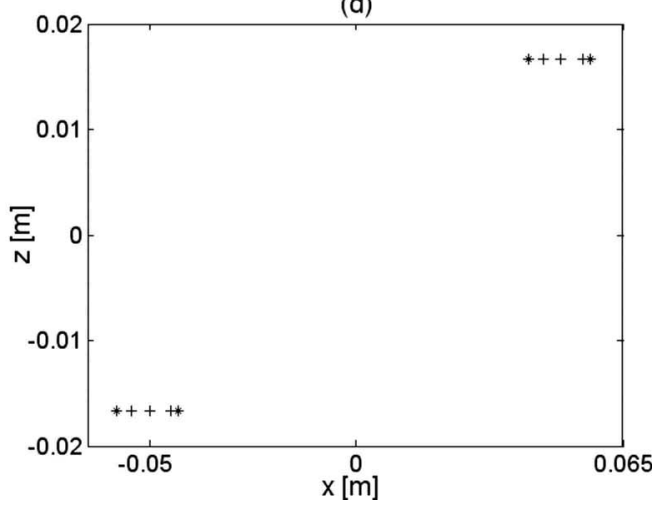

FIG. 5. Location and rms amplitude of monopoles consist of multipoles used for simulation at 1000 and $3000 \mathrm{~Hz}$, where "+" indicates amplitude of positive 1 and "*" indicates amplitude of negative 1. (a) $1000 \mathrm{~Hz}, x y$-coordinate; (b) $3000 \mathrm{~Hz}, x y$-coordinate; (c) $1000 \mathrm{~Hz}, x z$-coordinate; (d) $3000 \mathrm{~Hz}, x z$-coordinate.

located off the $x$-axis, these are $(-0.15,0,-0.05)$, $(0.15,0,0.05) \mathrm{m}$ for $1000 \mathrm{~Hz}$ and $(-0.05,0,-0.0167)$, $(0.05,0,0.0167) \mathrm{m}$ for $3000 \mathrm{~Hz}$.

The normalized amplitude of multipole source beamforming intensity estimated at the $z=0$ plane using sound pressure measurements at $z=0.1 \mathrm{~m}$ and $z=0.05 \mathrm{~m}$ and frequencies of 1000 and $3000 \mathrm{~Hz}$ are shown in Figs. 6 and 7. The order of near-field MVDR BF for both frequencies was $n=1$, which corresponds to the array manifold vector for spherical wave propagation. The hypothesized source surfaces to estimate near-field MVDR weights are located at $z$ $=0 \mathrm{~m}$ and $z=0.02 \mathrm{~m}$ for frequencies of 1000 and $3000 \mathrm{~Hz}$, respectively. The normalized beamforming intensity level away from the actual source region for first order inversely weighted beamforming is lower than that of uniformly weighted CBF. So the first order inversely weighted beamforming procedure is more accurate than uniformly weighted CBF for resolving closely located sound sources both at 1000 and $3000 \mathrm{~Hz}$. As the order of inversely weighted beamforming is increased, the intensity level away from the actual source region is decreased. However the intensity level of one of the sources located further away from the measurement surface is also decreased as the order of inversely weighted beamforming is increased. The intensity level of third order inversely weighted beamforming in the vicinity of the source located further away from the measurement surface is significantly lower than that of uniformly weighted $\mathrm{CBF}$ or first order inversely weighted beamforming. The intensity level away from the sources is not significantly lower than that of first order inversely weighted beamforming. So for both frequencies, first or second order inversely weighted beamforming performs best for source localization and visualization of nonreverberant multipole sources among IWBF procedures considered in this section.

The next algorithm considered was the optimized weight algorithm. For this algorithm, the optimized weights to minimize the maximum side lobe level were calculated under the assumption that the monopole source was located at the coordinate origin. Since weights are optimized for the monopole source located at the coordinate origin, beamforming intensity estimated at $3000 \mathrm{~Hz}$ represents the location of sources better than that estimated at $1000 \mathrm{~Hz}$, probably due to the fact that the source location at $3000 \mathrm{~Hz}$ is closer to the coordinate origin than that at $1000 \mathrm{~Hz}$.

The results in Figs. 6 and 7 show that the near-field MVDR beamforming intensity level estimated from nearfield measurements provides the clearest image in the source region. The amplitude of beamforming intensity represented as $|\mathrm{In}|$ in the figures and captions is estimated using CBF, IWBF with different orders, optimized weight beamforming, and MVDR. However, second order inversely weighted beamforming provides the lower beamforming intensity level outside of the source region compared to that of nearfield MVDR beamforming. Among the beamforming procedures considered in the present work, near-field MVDR beamforming appears to give the best sound source visualization for the slightly out-of-plane multipole simulation. 

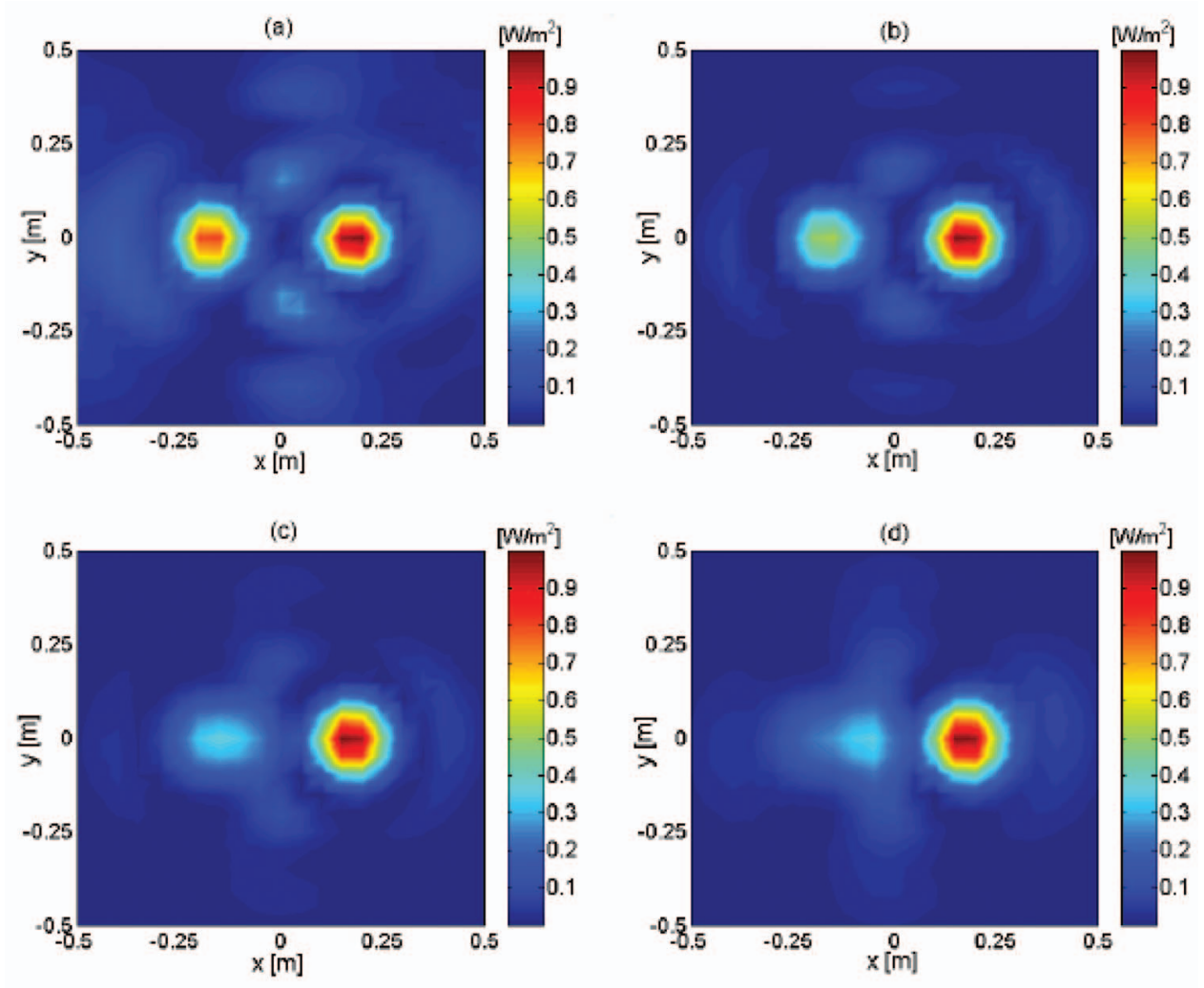

FIG. 6. Normalized amplitude of estimated beamforming multipole intensity based on anechoic measurement at $1000 \mathrm{~Hz}, z=0.1 \mathrm{~m}$ using planar array. (a) CBF $|\operatorname{In}|, z=0$; (b) IWBF $1 / \mathrm{R}, \mathrm{BF}$ $|\operatorname{In}|, z=0$; (c) IWBF $1 / \mathrm{R}^{2}, \mathrm{BF}|\mathrm{In}|, z$ $=0$; (d) $\operatorname{IWBF} 1 / \mathrm{R}^{3}, \mathrm{BF}|\operatorname{In}|, z=0$; (e) optimized weights, $\mathrm{BF}|\mathrm{In}|, z=0$; (f) MVDR, BF $|\mathrm{In}|, z=0$.
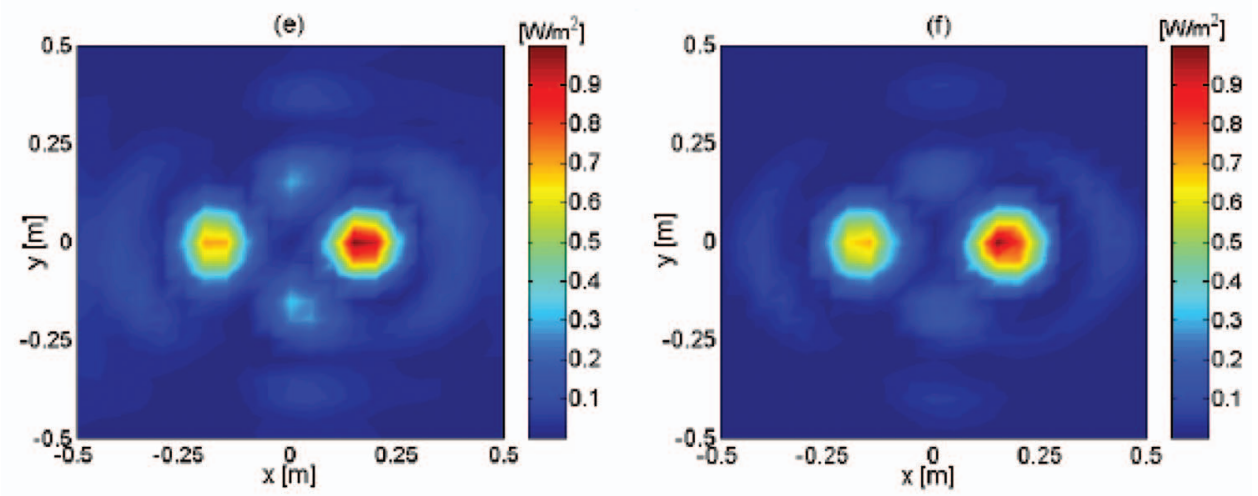

\section{B. Reverberant multipole simulation with two- dimensional array}

In Sec. III A, the relative performances of the various near-field beamforming procedures were compared for the out-of-plane multipoles based on noiseless anechoic measurements. In this section, the multipole simulation is performed in a reverberant environment with added random measurement noise. The method of images of sources was used to generate the sound pressure with reverberation.

An identical multipole source and measurement geometry, as described in Sec. III A, is used for the multipole simulation in this section except that now two rigid surfaces are located normal to each other to simulate reverberation. The location of the rigid surfaces relative to the multipole sources is shown in Fig. 8. The reverberant-to-direct energy ratio of the measurement pressure for the multipole simulation is estimated as $6.5 \%$ and $5.3 \%$ for 1000 and $3000 \mathrm{~Hz}$, respectively. Also the root-mean-square (rms) error between the directly measured pressure with and without reverbera- tion is estimated as $25.5 \%$ and $23.1 \%$ for 1000 and $3000 \mathrm{~Hz}$, respectively. Since the measurement surface is located close to the source, the reverberant-to-direct energy ratio is small although the rms error between the directly measured pressure with and without reverberation is not small. However, the dimension of measurement geometry shown in Fig. 8 represents pressure measurement in a practical reverberant measurement environment well.

The normalized amplitude of multipole beamforming intensity estimated using MVDR and optimized weights from measurements with reverberation and $20 \mathrm{~dB}$ additive random noise at 1000 and $3000 \mathrm{~Hz}$ is shown in Fig. 9. By comparing the amplitude of beamforming intensity estimated using both anechoic and reverberant pressure measurements with and without $20 \mathrm{~dB}$ additive random noise, it is observed that beamforming intensity estimates are very similar. It is also true for the results of IWBF, which is not shown in the present work. So near-field beamforming procedures with 

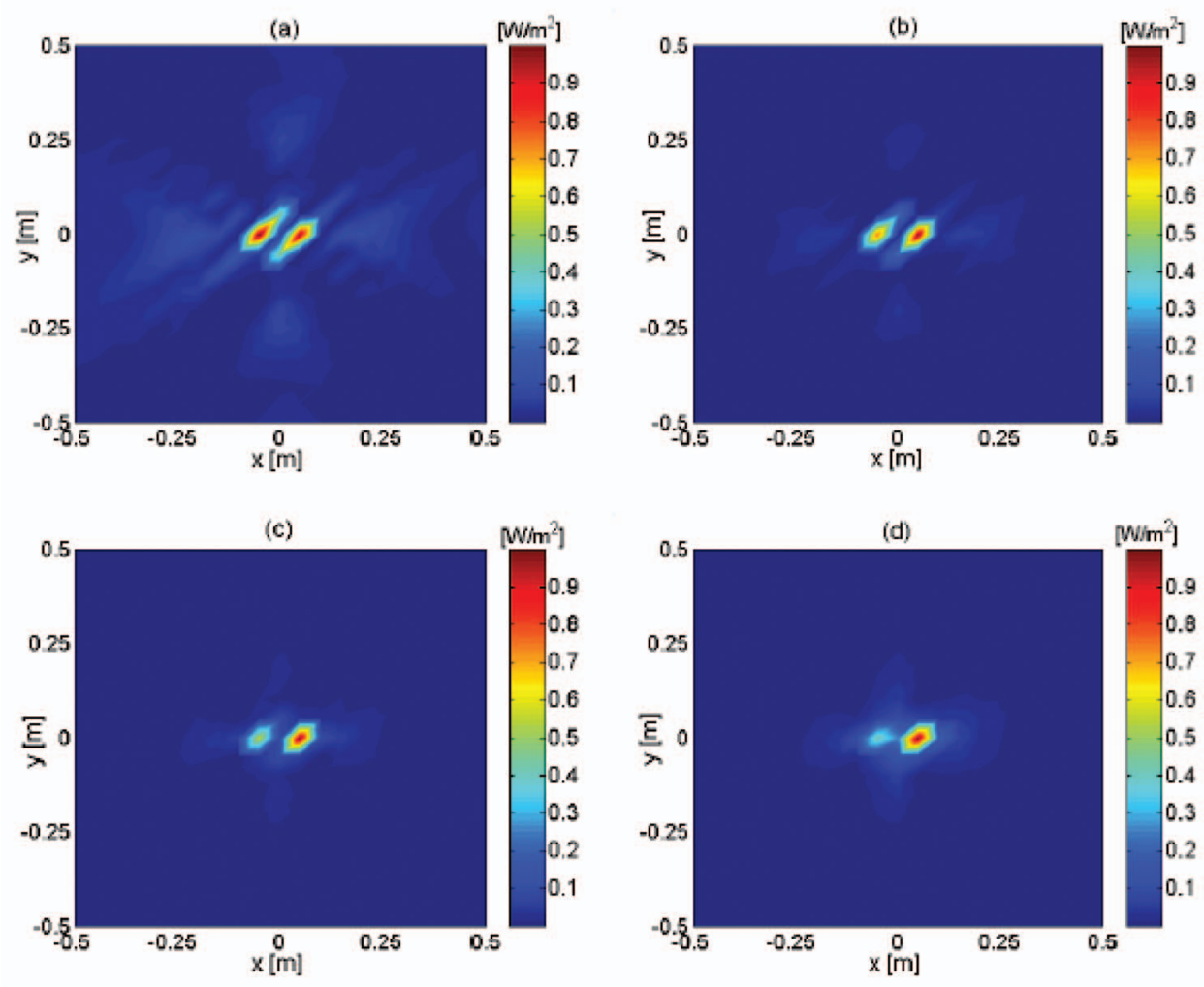

FIG. 7. Normalized amplitude of estimated beamforming multipole intensity based on anechoic measurement at $3000 \mathrm{~Hz}, z=0.05 \mathrm{~m}$ using planar array. (a) CBF $|\operatorname{In}|, z=0$; (b) IWBF $1 / \mathrm{R}$, $\mathrm{BF}|\mathrm{In}|, z=0$; (c) IWBF $1 / \mathrm{R}^{2}, \mathrm{BF}|\mathrm{In}|$, $z=0$; (d) IWBF $1 / \mathrm{R}^{3}, \mathrm{BF}|\operatorname{In}|, z=0$; (e) optimized weights, BF $|\operatorname{In}|, z=0$; (f) MVDR, BF $|\mathrm{In}|, z=0$.
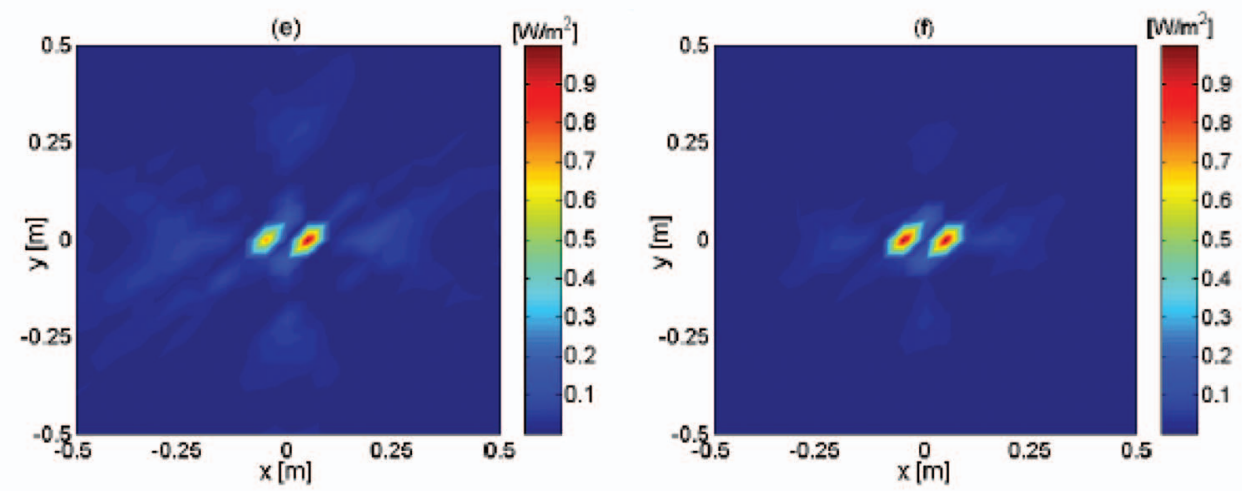

different weighting strategies are effective both for anechoic and reverberant pressure measurements with random measurement noise.

\section{ENCLOSED LOUDSPEAKER MEASUREMENT}

The results presented in Sec. III were based on numerical simulations. In this section, experimental results are pre-

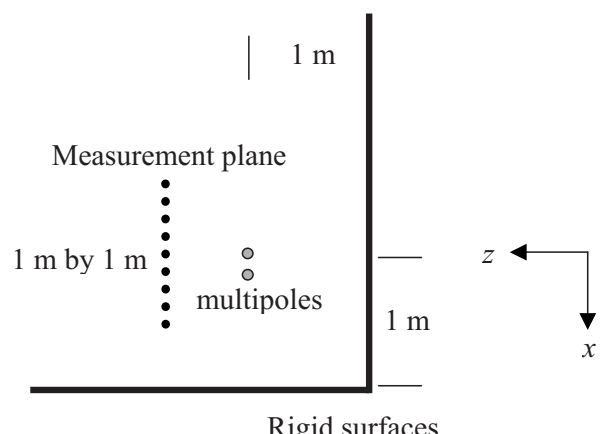

FIG. 8. Location of rigid surfaces relative to multipole sources to simulate reverberation. The centroid of multipoles is located at the coordinate origin.

sented using an enclosed loudspeaker measurement setup. First, the loudspeaker measurement geometry and experimental apparatus are described in Sec. IV A. Then, in Sec. IV B, IWBF, optimized weight beamforming, and near-field MVDR beamforming intensity estimates using a planar measurement surface are compared.

\section{A. Enclosed loudspeaker measurement description}

The near-field measurement geometry for the enclosed loudspeaker experiment is shown in Fig. 10 . A $12.7 \mathrm{~cm}$ diameter loudspeaker mounted in an enclosure was used as the source. All measurements were done in an anechoic chamber. The actual loudspeaker and enclosure are shown in Fig. 11. The outer surface of the loudspeaker is on the same plane with the surface of the enclosure. The measurement plane was $2 \mathrm{~cm}$ above the surface of the enclosure. An array of 11 microphones was used to take simultaneous measurements in the $x$-direction. The array was then moved in increments of $2 \mathrm{~cm}$ in the $y$-direction to take $16 y$-direction measurements. This resulted in a $22 \times 32 \mathrm{~cm}^{2}$ rectangular measurement sur- 


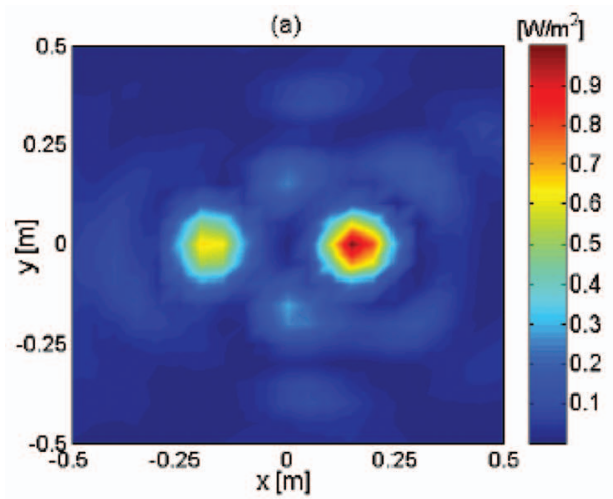

(c)

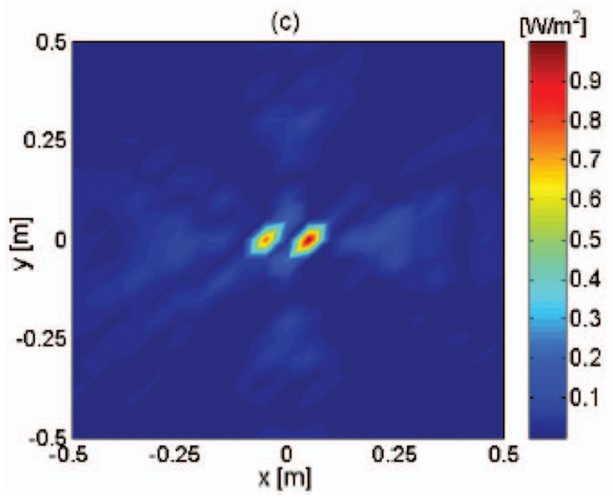

(b)

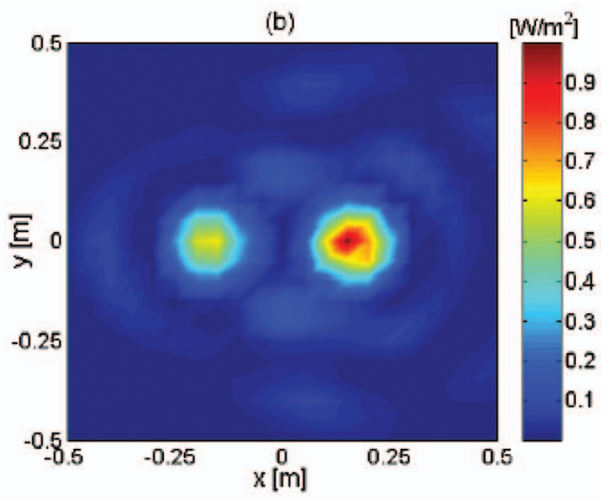

(d)

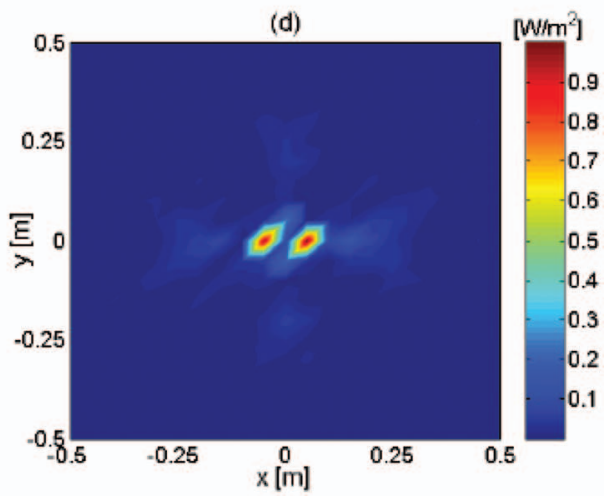

FIG. 9. Normalized amplitude of estimated beamforming multipole intensity at $z=0$ from measurement with reverberation and $20 \mathrm{~dB}$ additive random noise at $1000 \mathrm{~Hz}, z=0.1 \mathrm{~m}$ and $3000 \mathrm{~Hz}, z=0.05 \mathrm{~m}$ using planar array. (a) Optimized weights, BF $|\mathrm{In}|$, $1000 \mathrm{~Hz}$; (b) MVDR, BF $|\mathrm{In}|$, $1000 \mathrm{~Hz}$; (c) optimized weights, BF $|\mathrm{In}|, 3000 \mathrm{~Hz}$; (d) MVDR, BF $|\mathrm{In}|$, $3000 \mathrm{~Hz}$. face. The center of both the measurement surface and the source were on the $z$-axis. The loudspeaker enclosure surface was a $17.3 \times 26.3 \mathrm{~cm}^{2}$ rectangle and the center of the top surface of the enclosure coincided with the coordinate system origin.

The measurement system consisted of a National Instruments (NI) CompactDAQ chassis, NI cDAQ-9172, and NI 9233 signal conditioner, and a Dell Inspiron $640 \mathrm{~m}$ laptop computer was used to run the NI LABVIEW 8.2 data acquisition software. Eleven array microphones (G.R.A.S. Sound \& Vibration Type 40 PR) were used to make the sound pressure measurements: they are also shown in Fig. 11. The array microphones were calibrated using a B\&K Type 4230 microphone calibrator.

A random signal with a cutoff frequency of $6 \mathrm{kHz}$ was computer generated and played through a JBL power amplifier model 6260. The output of the JBL power amplifier was directly provided as input to the loudspeaker. Also the computer generated random signal was fed directly to the NI 9233 signal conditioner as the reference signal.

Field microphone signals are sampled at $20 \mathrm{kHz}$ with LABVIEW. A $0.25 \mathrm{~s}$ long Hanning window was applied to each temporal data record. The low pass filtered signals with

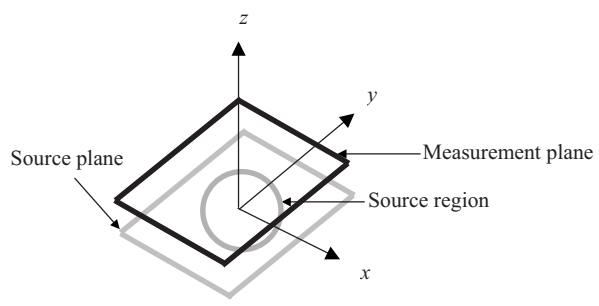

FIG. 10. Enclosed loudspeaker near-field measurement geometry definition. cutoff frequency of $8 \mathrm{kHz}$ were fast Fourier transformed and were averaged 119 times with $50 \%$ overlap and $4 \mathrm{~Hz}$ resolution to estimate the required transfer functions between reference and field microphone signals. The transfer functions between reference and field microphone signals were considered as measurement pressure in the present work.

\section{B. Near-field measurement results}

The spatial rms amplitude of the near-field sound pressure measurement of the enclosed loudspeaker is shown in

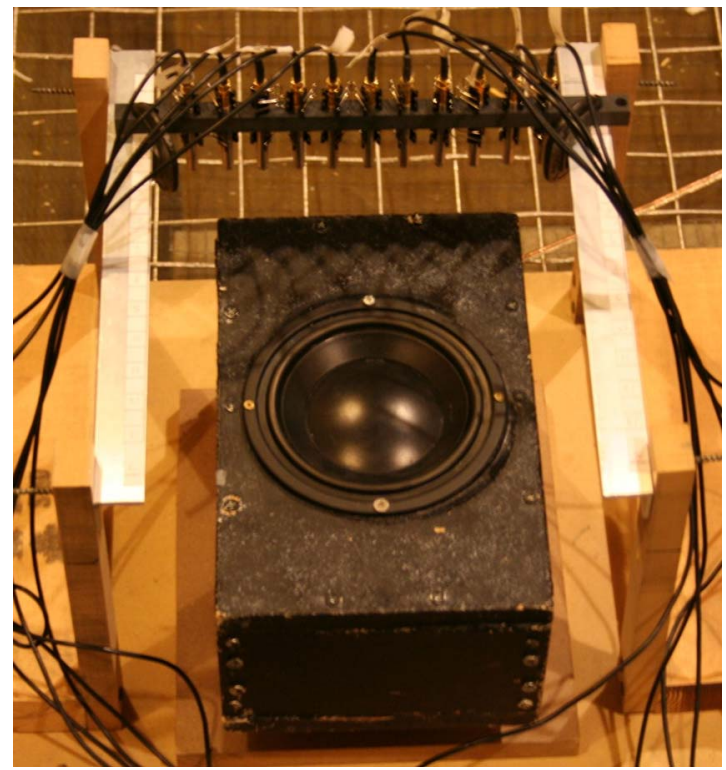

FIG. 11. (Color online) Enclosed loudspeaker source and microphone array for near-field measurement. 


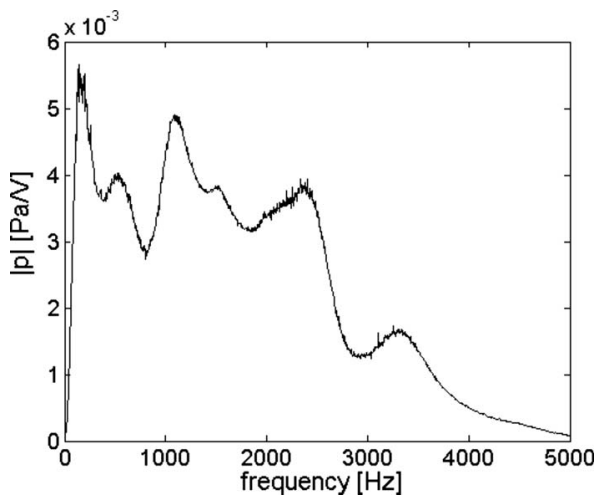

FIG. 12. Spatial rms of near-field pressure measurement.

Fig. 12. IWBF and near-field MVDR beamforming were implemented to operate at frequencies corresponding to the major peaks and dips of the spatial rms amplitude of the near-field sound pressure measurement. Specifically, four frequencies $(804,1088,2928$, and $3312 \mathrm{~Hz}$ ) were selected from Fig. 12 and the corresponding measurement pressure is shown in Fig. 13. These frequencies correspond to interesting mode shapes of the loudspeaker. 804 and $1088 \mathrm{~Hz}$ are modes where the entire surface of the loudspeaker moves in phase. At $2928 \mathrm{~Hz}$, a nodal line appears diagonally across the loudspeaker. Lastly, at $3312 \mathrm{~Hz}$, a mode exists where the center of speaker moves out of phase with the surrounding cone.

The magnitudes of the frequency response functions (the $H_{1}$ transfer functions between the response of the microphones and the input of the JBL amplifier) representing the measurement pressure amplitudes for the enclosed loudspeaker are shown in Fig. 12. Also BF intensity estimates $2.5 \mathrm{~cm}$ below the measurement surface or $0.5 \mathrm{~cm}$ below the loudspeaker enclosure surface using IWBF, optimized weight BF, and near-field MVDR BF are shown in Figs. 14 and 15. The order of IWBF, $n=2$ is used for all frequencies. For near-field MVDR BF, $n=1$ is used for 2928 and $3312 \mathrm{~Hz}$ and $n=2$ is used for 804 and $1088 \mathrm{~Hz}$. This was done because MVDR BF, $n=2$, removes measurement noise better than MVDR BF, $n=1$, especially at low frequencies. However, using MVDR BF, $n=1$, provides more detailed information about the source at higher frequencies. Optimized weights are estimated based on a monopole source located $0.5 \mathrm{~cm}$ below the coordinate origin and the actual size of the loudspeaker. Both BF intensity estimates at 804 and $1088 \mathrm{~Hz}$ are similar in terms of the shape of source even though $804 \mathrm{~Hz}$ is one of the lowest dips and $1088 \mathrm{~Hz}$ is one of the highest peaks in spatial rms of measurement pressure. Although not shown in the results, the shape of the source at frequencies below $2336 \mathrm{~Hz}$ is typically the same as that approximated from the $\mathrm{BF}$ intensity estimates at 804 and $1088 \mathrm{~Hz}$. The size of the source approximated from BF intensity estimates at 804 and $1088 \mathrm{~Hz}$ represents the actual size of the loudspeaker very well.

The BF intensity estimate of the enclosed loudspeaker measurement using near-field MVDR BF and higher order IWBF is very similar over the range of frequencies, except that measurement noise is removed relatively well in the intensity estimate using either higher order IWBF or nearfield MVDR BF. This is not shown in the present work. The
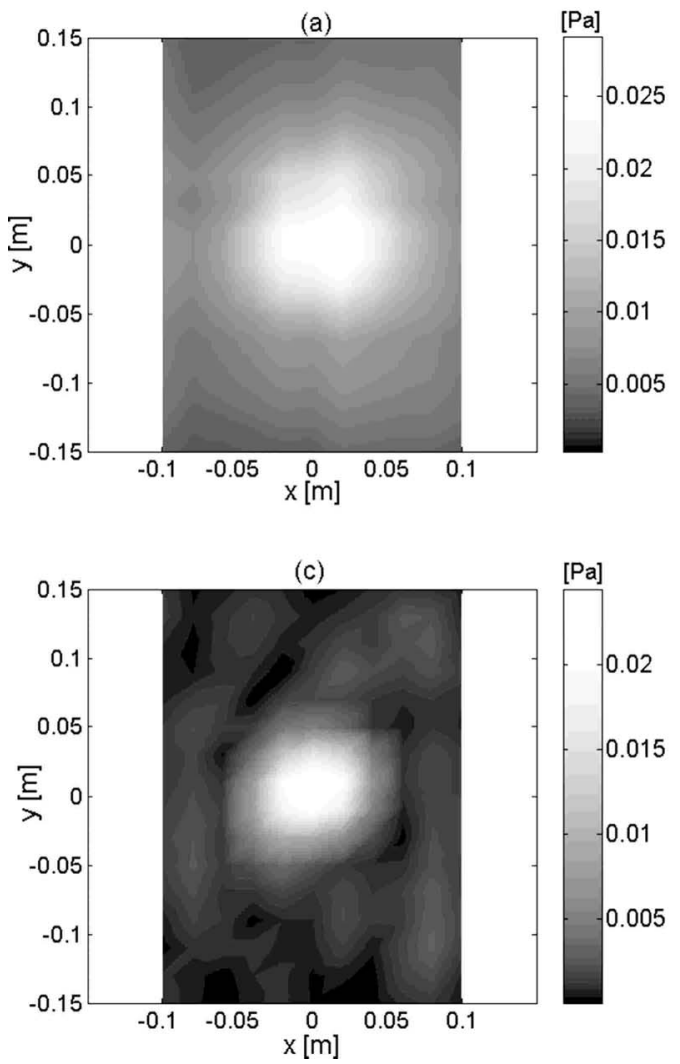

(b)

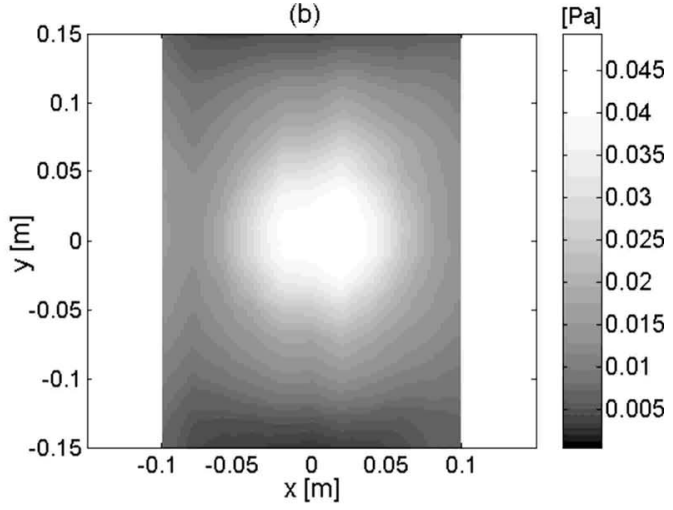

(d)

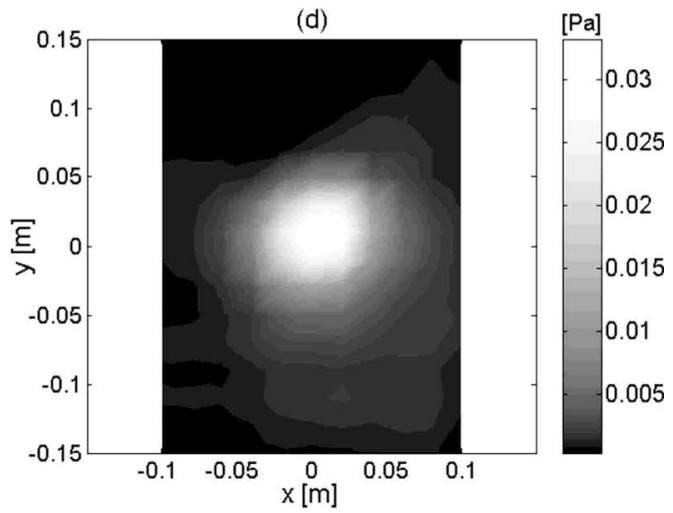

FIG. 13. Enclosed loudspeaker measurement pressure. (a) $|p|, 804 \mathrm{~Hz}$; (b) $|p|, 1088 \mathrm{~Hz}$; (c) $|p|, 2928 \mathrm{~Hz}$; (d) $|p|, 3312 \mathrm{~Hz}$. 
(a)

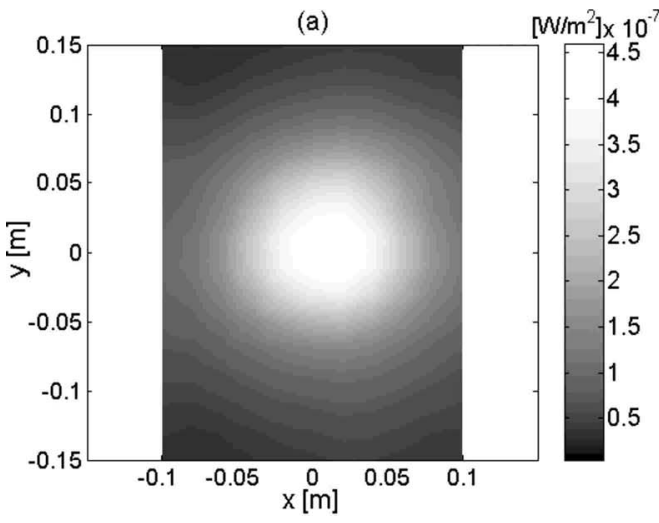

(c)
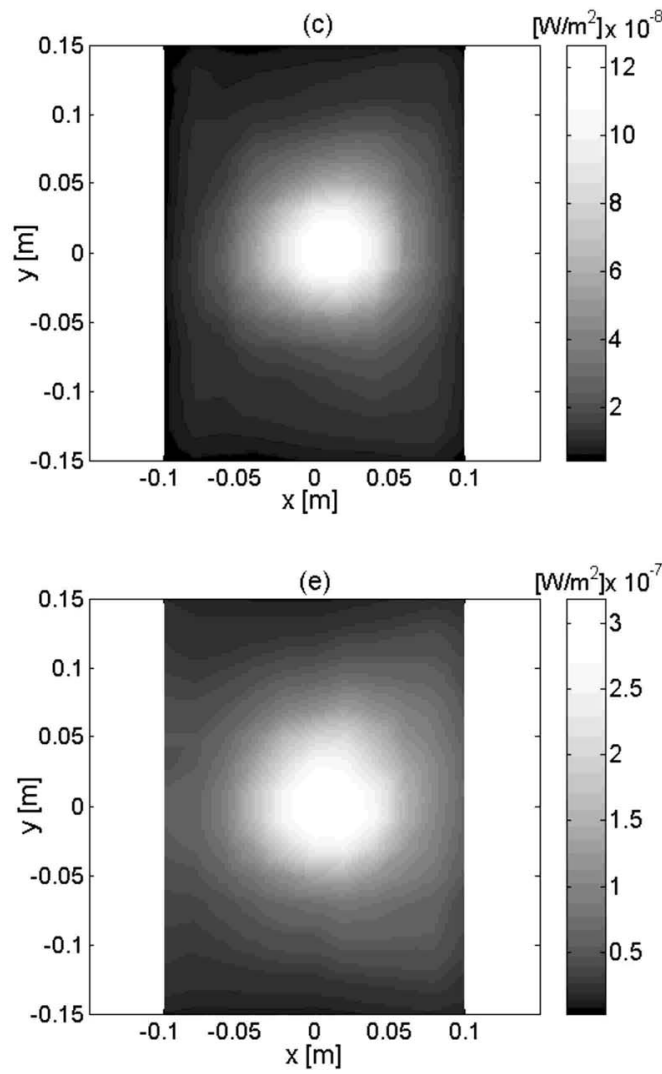

(b)

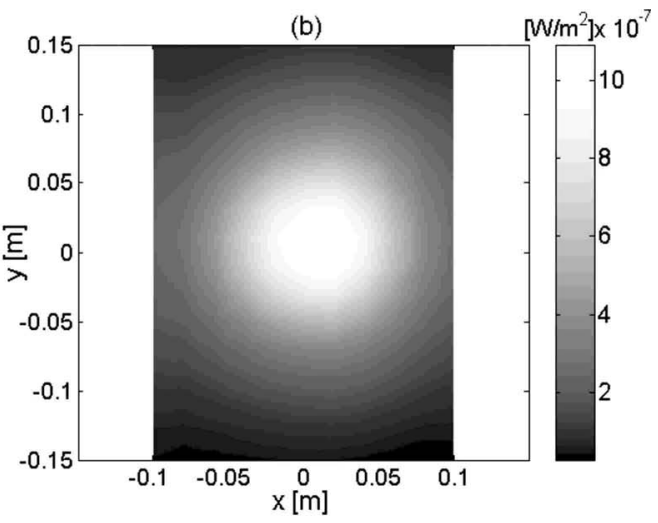

(d)
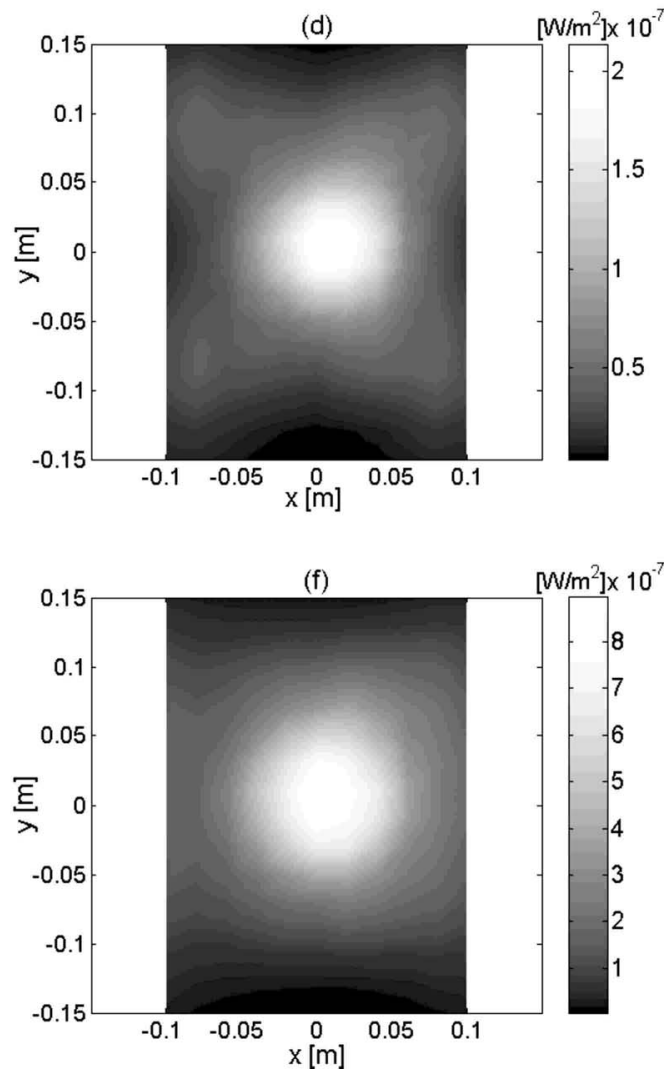

FIG. 14. Beamformed intensity of enclosed loudspeaker measurement. (a) IWBF |In|, $804 \mathrm{~Hz}$; (b) IWBF |In|, $1088 \mathrm{~Hz}$; (c) optimized weights, BF |In|, $804 \mathrm{~Hz}$; (d) optimized weight, BF |In|, 1088 Hz; (e) MVDR BF |In|, 804 Hz; (f) MVDR BF |In|, 1088 Hz.

shape of the source approximated from the BF intensity estimate is very similar for frequencies of $2336 \mathrm{~Hz}$ or below. However, both BF intensity estimates at 2928 and $3312 \mathrm{~Hz}$ are quite different from those at other frequencies. The lowest dip in spatial rms of pressure measurement is $2928 \mathrm{~Hz}$, and the highest peak in spatial rms of pressure measurements above $3 \mathrm{kHz}$ is $3312 \mathrm{~Hz}$. It appears that near-field MVDR $\mathrm{BF}$ intensity provides more detailed information about the source than IWBF or optimized weight BF. The nodal line in BF intensity at $2928 \mathrm{~Hz}$ using near-field MVDR BF shown in Fig. 15(e) indicates that the even mode dominates at this frequency. Since even modes are very inefficient sound radiators $^{27}$ and $2928 \mathrm{~Hz}$ corresponds to the lowest dip in the spatial rms of measurement pressure, the even mode shape at this frequency is reasonable. At $3312 \mathrm{~Hz}$, the BF intensity estimates using IWBF and near-field MVDR BF are similar in that it can be seen that the center of the loudspeaker radiates sound at the highest amplitude. Overall, the size of the source approximated using IWBF, optimized weight BF, and near-field MVDR BF intensity is reasonable compared to the actual size of the loudspeaker.

\section{CONCLUSIONS}

In the present work, fixed and adaptive beamforming algorithms are modified to provide very effective acoustic source imaging capabilities using near-field measurements. Near-field beamforming weightings are estimated based on spherical wave array manifold vectors with spatial windows. To show this, both simulations and experiments were done for complex sound sources. The improved source resolution accuracy is accomplished by application of different weight- 

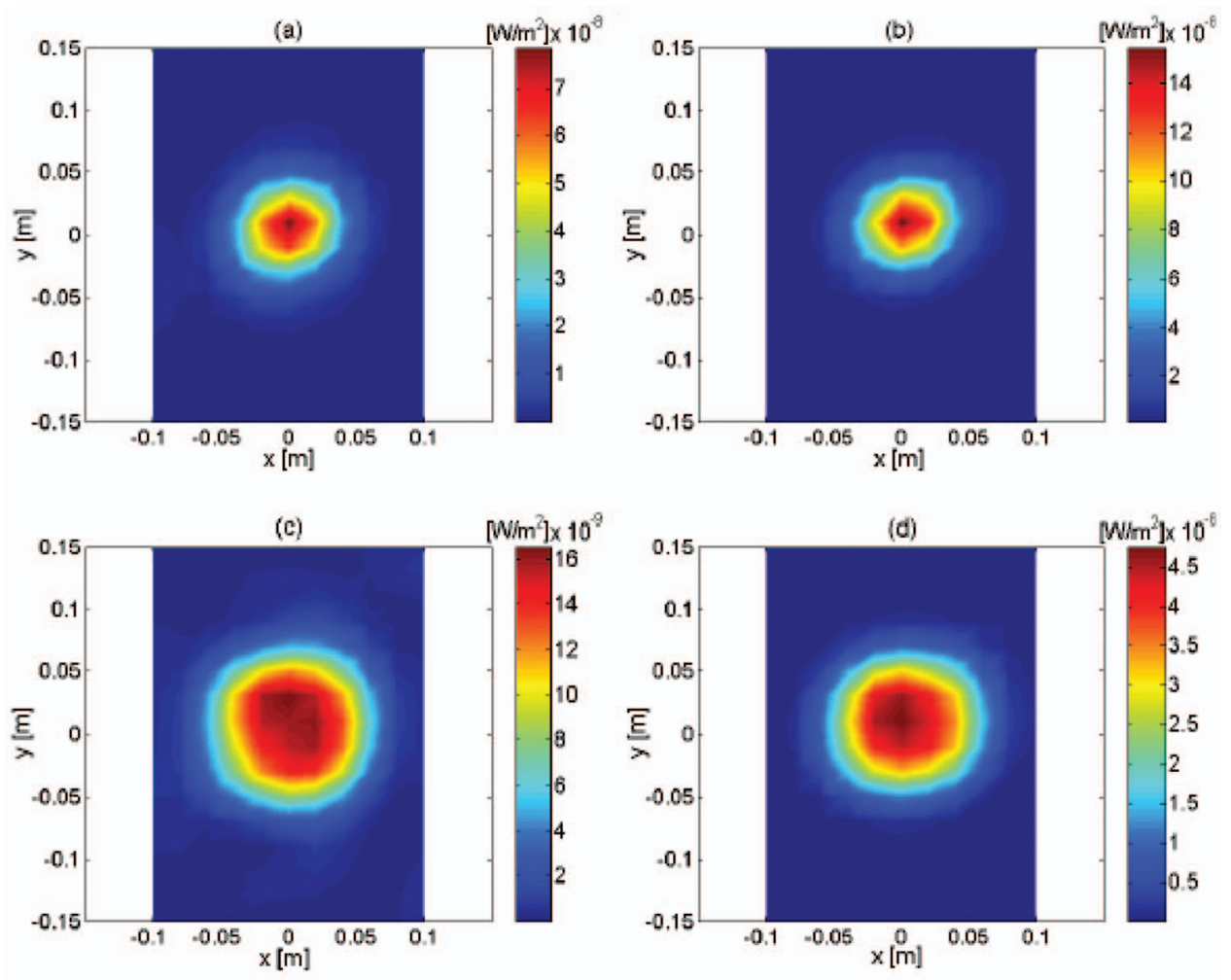

FIG. 15. Beamformed intensity of enclosed loudspeaker measurement. (a) IWBF |In|, $2928 \mathrm{~Hz}$; (b)IWBF |In|, $3312 \mathrm{~Hz}$; (c) optimized weights, BF |In|, $2928 \mathrm{~Hz}$; (d) optimized weight, BF |In|, 3312 Hz; (e) MVDR BF |In|, $2928 \mathrm{~Hz}$; (f) MVDR BF |In|, $3312 \mathrm{~Hz}$.

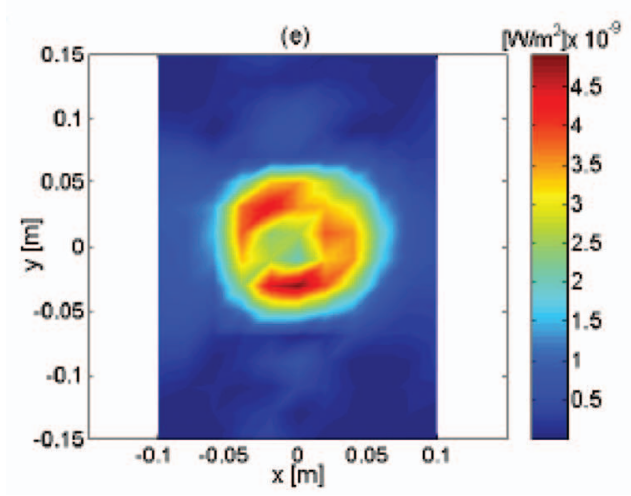

ing strategies, including higher order inversely proportional weighting, optimized weights to reduce side lobe level, and near-field phase corrected MVDR beamforming with spherical wave array manifold vectors with spatial windows.

The numerical simulation results show that using higher order inversely proportional weighting $(n>2)$ does not necessarily improve the source resolution accuracy. The optimized weight algorithm provides superior performance (in terms of source resolution accuracy) only when true source location is known. However, source resolution accuracy degrades when the weights are optimized for unknown or inaccurate source location.

Source resolution accuracy of the standard MVDR beamformer using near-field measurement is not as good as beamforming with first order inversely proportional weights. However, in this work a near-field phase corrected version of MVDR with spherical wave array manifold vectors with spatial windows is introduced and provides significantly improved source resolution accuracy. This new near-field MVDR beamformer is accurate for visualization of sources based on near-field measurements with and without reverberation and random measurement noise.
Multipole simulation were performed both with and without reverberation and additive random measurement noise. Using high-resolution near-field beamforming procedures significantly removes both $20 \mathrm{~dB}$ additive random noise in the pressure measurements and reverberation created by two infinite rigid surfaces. In addition, near-field sound pressure of enclosed loudspeaker is measured, and the source is visualized using higher order IWBF, optimized weight BF, and near-field MVDR BF procedures. Both IWBF and nearfield MVDR BF procedures provided similar results except at $2928 \mathrm{~Hz}$, which is the lowest dip of the spatial rms of measurement pressure. More detailed visualization of the source is provided by near-field MVDR BF intensity compared to that provided by IWBF or optimized weight BF at $2928 \mathrm{~Hz}$. Overall, it can be concluded that near-field MVDR beamforming and higher order inversely proportional weights with spatial windows can be implemented to visualize sound sources more accurately than $\mathrm{CBF}$ with other weighting strategies for various near-field sound pressure measurement environments. 
${ }^{1}$ H. L. Van Trees, Optimum Array Processing: Part IV of Detection, Estimation, and Modulation (Wiley, New York, 2002).

${ }^{2}$ J. Billingsley and R. Kinns, "The acoustic telescope," J. Sound Vib. 48, 485-510 (1976).

${ }^{3}$ P. T. Soderman and S. C. Noble, "Directional microphone array for acoustic studies of wind tunnel models," J. Aircr. 12, 168-173 (1975).

${ }^{4}$ T. Suzuki, "Identification of multipole noise sources in low Mach number jets near the peak frequency," J. Acoust. Soc. Am. 119, 3649-3659 (2006). ${ }^{5}$ H. Kook, G. B. Moebs, P. Davies, and J. S. Bolton, "An efficient procedure for visualizing the sound field radiated by vehicle during standardized passby tests," J. Sound Vib. 233, 137-156 (2000).

${ }^{6} \mathrm{C}$. L. Dolph, "A current distribution for broadside arrays which optimizes the relationship between beam width and side-lobe level," Proc. IRE 34, 335-348 (1946).

${ }^{7}$ J. Capon, "High-resolution frequency-wavenumber spectrum analysis," Proc. IEEE 57, 1408-1418 (1969).

${ }^{8}$ B. G. Ferguson, "Minimum variance distortionless response beam forming of acoustic array data," J. Acoust. Soc. Am. 104, 947-954 (1998).

${ }^{9} \mathrm{~S}$. Holm and B. Elgetun, "Optimization of the beam pattern of 2D sparse arrays by weighting," Proc.-IEEE Ultrason. Symp. 1995, 1345-1348.

${ }^{10} \mathrm{~S}$. Holm, B. Elgetun, and G. Dahl, "Properties of the beampattern of weight- and layout-optimized sparse arrays," IEEE Trans. Ultrason. Ferroelectr. Freq. Control 44, 983-991 (1997).

${ }^{11}$ Y. T. Cho, M. J. Roan, and J. S. Bolton, "A comparison of beam forming and acoustical holography for sound source visualization," Proc. Inst. Mech. Eng., Part C: J. Mech. Eng. Sci. (In press, 2009).

${ }^{12}$ J. D. Maynard, E. G. Williams, and Y. Lee, "Nearfield acoustic holography: I. Theory of generalized holography and development of NAH," J. Acoust. Soc. Am. 78, 1395-1413 (1985).

${ }^{13}$ E. G. Williams, H. D. Dardy, and K. B. Washburn, "Generalized near-field acoustic holography for cylindrical geometry: Theory and experiment," J. Acoust. Soc. Am. 81, 389-407 (1987).

${ }^{14}$ E. G. Williams, Fourier Acoustics: Sound Radiation and Near-Field Acoustical Holography (Academic, London, UK, 1999).

${ }^{15}$ R. Steiner and J. Hald, "Near-field acoustical holography without the errors and limitations caused by the use of spatial DFT," Proceedings of
ICSV6 (1999), pp. 843-850.

${ }^{16} \mathrm{~J}$. Hald, "Patch near-field acoustical holography using a new statistically optimal method," Proceedings of INTER-NOISE 2003, pp. 2203-2210 (2003).

${ }^{17}$ Y. T. Cho, J. S. Bolton, and J. Hald, "Source visualization by using statistically optimized near-field acoustical holography in cylindrical coordinates," J. Acoust. Soc. Am. 118, 2355-2365 (2005).

${ }^{18} \mathrm{H}$. Schjær-Jacobsen and K. Madsen, "Synthesis of nonuniformly spaced arrays using a general nonlinear minimax optimization method," IEEE Trans. Antennas Propag. 24, 501-506 (1976).

${ }^{19}$ P. Jarske, T. Saramäki, S. K. Mitra, and Y. Neuvo, "On properties and design of nonuniformly spaced linear arrays," IEEE Trans. Acoust., Speech, Signal Process. 36, 372-380 (1988).

${ }^{20}$ R. M. Leahy and B. D. Jeff, "On the design of maximally sparse beam forming arrays," IEEE Trans. Antennas Propag. 39, 1178-1187 (1991).

${ }^{21}$ D. H. Turnbull and F. S. Foster, "Beam steering with pulsed twodimensional transducer arrays," IEEE Trans. Ultrason. Ferroelectr. Freq. Control 38, 320-333 (1991).

${ }^{22}$ P. K. Weber, R. M. Schmitt, B. D. Tylkowski, and J. Steck, "Optimization of random sparse 2-D transducer arrays for 3-D electronic beam sterring and focusing," Proc.-IEEE Ultrason. Symp. 1994, 1503-1506.

${ }^{23}$ R. E. Davidsen, J. A. Jensen, and S. W. Smith, "Two dimensional random arrays for real time volumetric imaging," Ultrason. Imaging 16, 143-163 (1994).

${ }^{24}$ G. R. Lockwood, P. Li, M. O'Donnell, and F. S. Foster, "Optimizing the radiation pattern of sparse periodic linear arrays," IEEE Trans. Ultrason. Ferroelectr. Freq. Control 43, 7-14 (1996).

${ }^{25}$ G. R. Lockwood and F. S. Foster, "Optimizing the radiation pattern of sparse periodic two-dimensional arrays," IEEE Trans. Ultrason. Ferroelectr. Freq. Control 43, 15-19 (1996).

${ }^{26}$ V. Murino, A. Trucco, and C. S. Regazzoni, "Synthesis of unequally spaced arrays by simulated annealing," IEEE Trans. Signal Process. 44, 119-123 (1996).

${ }^{27}$ F. Fahy, Sound and Structural Vibration: Radiation, Transmission and Response (Academic, London, UK, 1985). 